\title{
Field and temperature induced effects in the surface modification process
}

\author{
Sz. Csonka, A. Halbritter, and G. Mihály \\ Electron Transport Research Group of the Hungarian Academy of Science at Budapest University \\ of Technology and Economics, 1111 Budapest, Hungary
}

E. Jurdik, O. I. Shklyarevskii, ${ }^{\text {a) }}$ S. Speller, and H. van Kempen

NSRIM, University of Nijmegen, Toernooiveld 1, 6525 ED Nijmegen, The Netherlands

(Received 27 April 2004; accepted 24 August 2004)

\begin{abstract}
We used the mechanically controllable break junction technique to discriminate between the electric field and temperature effects in the process of surface modification. The electric field strength at the surface of electrodes was accurately determined using the field emission resonance spectra and was gradually raised to the point where surface modification starts. We found that only a limited number of metals with large values of the work function, $\gtrsim 4.5-5 \mathrm{eV}$, and evaporation fields, $\lesssim 1.5-2 \mathrm{~V} / \AA$, can be modified in this way. Adsorption of He on the surface drastically increases the local work function of material and enables the field induced nanostructuring of the electrode surface practically for all metals. (C) 2004 American Institute of Physics. [DOI: 10.1063/1.1807521]
\end{abstract}

\section{INTRODUCTION}

In a standard operational mode of scanning tunneling microscopes (STM) the interaction between the tip and the sample under investigation is presumed to be negligibly small. ${ }^{1}$ However, this interaction cannot be ignored at close electrode separations and/or high bias voltages applied across the vacuum gap. Since the invention of STM numerous attempts were made to employ the tip-sample interaction as a tool for fabrication of nanostructures and controlled modification of the sample surface. ${ }^{2}$ In the majority of cases short voltage pulses with an amplitude of up to $10 \mathrm{~V}$ were used to produce different types of structures on an originally smooth surface (see Refs. 3 and 4 and references therein). Two different physical mechanisms were suggested to explain the experimental data. The first mechanism views the electric field induced effects-such as field desorption (evaporation) and field-enhanced surface diffusion of atoms - as the main reason of nanostructuring. ${ }^{4-7}$ The second mechanism assumes formation of a direct mechanical contact $^{8,9}$ at a certain stage of the modification process as a result of thermal expansion of the tip ${ }^{10}$ or field induced surface deformation of the electrodes. ${ }^{11}$ It is extremely difficult to discriminate between these field and temperature effects, especially in experiments with voltage pulses. This is because these effects are closely interconnected as the local overheating of the sample surface is an inseparable part of the modification process and occurs on approximately the same time scale. Not surprisingly, it was claimed in Ref. 3 that the modification mechanisms proposed so far "are not the physical mechanisms underlaying the structure formation."

In this paper we attempt to resolve the above issue by exploiting the advantages of the mechanically controllable

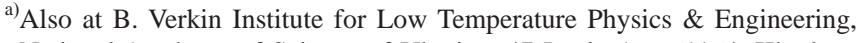
National Academy of Science of Ukraine, 47 Lenin Av., 61164, Kharkov, Ukraine.
}

break junction (MCBJ) technique and by using a fresh approach to the problem that is based on the following considerations.

(i) In most cases the surface modification experiments concentrated on finding the threshold amplitude of voltage pulses for initiation of the nanostructuring process. However, the crucial parameter of the process is the electric field strength $F$ at the electrode surfaces. This electric field can be accurately determined by measuring the field emission resonance (FER) spectra in the Fowler-Nordheim regime $(\mathrm{eV}$ $\gg \phi$ ), where $e$ is the electron charge, $V$ is the bias voltage, and $\phi$ is the work function of material. ${ }^{12}$

(ii) At low temperatures the thermal effects are reduced due to the smallness of the linear expansion coefficient. In addition, the perceptible elongation of the electrodes occurs on a millisecond scale and is proportional to the square root of time, $t^{1 / 2}$, because of the large values of the thermal diffusivity. ${ }^{13}$ Therefore, the distance $S$ between the electrodes of MCBJ can easily be controlled by a feedback circuit.

Finally, the experimental situation in our case is quasistationary, whereas modification with voltage pulses includes a number of ill-controllable transient processes.

(iii) We demonstrate that increasing the electric field above a critical value results in abrupt field evaporation/ desorption of a sizable amount of material of the MCBJ electrodes. Adsorption of $\mathrm{He}$ on the surface of electrodes increases dramatically the local work function of material and the field-related modification process occurs at an extremely small field emission current.

\section{EXPERIMENT}

Over the last decade the MCBJ technique was developed to a powerful tool for numerous applications and is described elsewhere. ${ }^{14}$ In our current experiments we used a slightly modified version of the traditional sample mounting and present it in Fig. 1. It includes two pieces $(5 \times 2.5$ $\times 1 \mathrm{~mm}^{3}$ ) of shear piezoceramic (that gives a horizontal displacement of its surface upon applied voltage) placed under- 

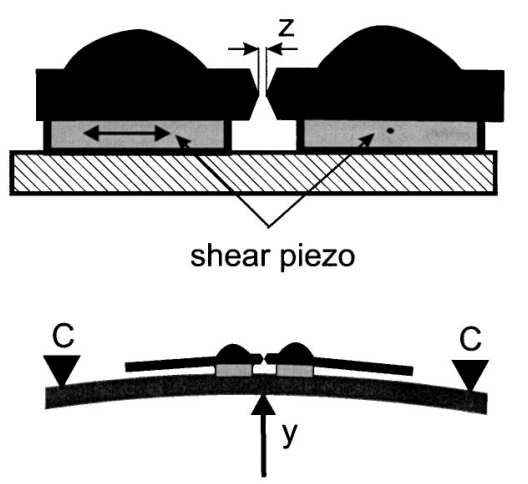

FIG. 1. Modified sample mounting. The wire is glued on top of a shear piezoceramic attached to a phosphor bronze bending beam. The distance between the electrodes is controlled by vertically pushing the bending beam at the center or by applying voltage on the left-hand side shear piezo. All distances are exaggerated for clarity.

neath the anchoring points of the sample wire. The righthand side piezo (moving in the plane normal to the picture) was used for two-dimensional scan of the electrode surface. The left-hand side piezo served for adjustment of the electrode separation when measuring the $S(V)$ dependencies. Also, the distance between the electrodes can be tuned by changing the substrate deflection. We found that this type of the sample mounting practically does not affect the junction stability. The electrode displacement $\Delta S$ was calibrated very accurately using the FER spectra measured in vacuum as proposed in Ref. 12. For piezoceramic in use (PXE 54) we found a conversion factor of $0.72 \pm 0.02 \AA / \mathrm{V}$ at $4.2 \mathrm{~K}$.

$\mathrm{Pt}, \mathrm{Au}$, and $\mathrm{W}$ wires with a diameter of $50-100 \mu \mathrm{m}$ as well as $25 \mu \mathrm{m}$ thick $/ 300 \mu \mathrm{m}$ wide Dy foil were used as sample materials. This selection of metals covers the $\phi$ values from $\sim 2.8 \mathrm{eV}$ for Dy to $\sim 5.5 \mathrm{eV}$ for Pt. The break of the notched samples and subsequent measurements were performed in a cryogenic ultrahigh vacuum (UHV) environment or in a He exchange gas at $4.2 \mathrm{~K}$. On some occasions, $S(V)$ dependencies were taken directly in liquid He in a temperature range of $1.2-4.2 \mathrm{~K}$.

The electrode separation $S$ as a function of the applied bias voltage $V$ was measured in a constant current mode using a set of current-to-voltage converters with a gain ranging from $1 \mathrm{~V} / \mathrm{nA}$ to $1 \mathrm{~V} / \mathrm{mA}$. Data were recorded with Keithley 2182 nanovoltmeters in a slow mode (5-30 points/s) and a voltage range from $1 \mu \mathrm{V}$ to $10 \mathrm{~V}$. Simultaneously, an AT-MIO-16XE-50 National Instruments data acquisition board was used in a fast mode (20000 points/s) in a 0.01-10 V range for time resolved measurements. The exceptional stability of MCBJ permits us to recover accurate FER spectra by numerical differentiation of $S(V)$ dependencies with no lock-in techniques required. ${ }^{15,16}$

All our results presented below are based on a careful analysis of few hundreds of $S(V)$ curves obtained for five to six different samples of every metal.

\section{RESULTS AND DISCUSSION}

\section{A. Electric field effects}

The distance-voltage characteristics taken in a constant current mode depend primarily on the metal work function

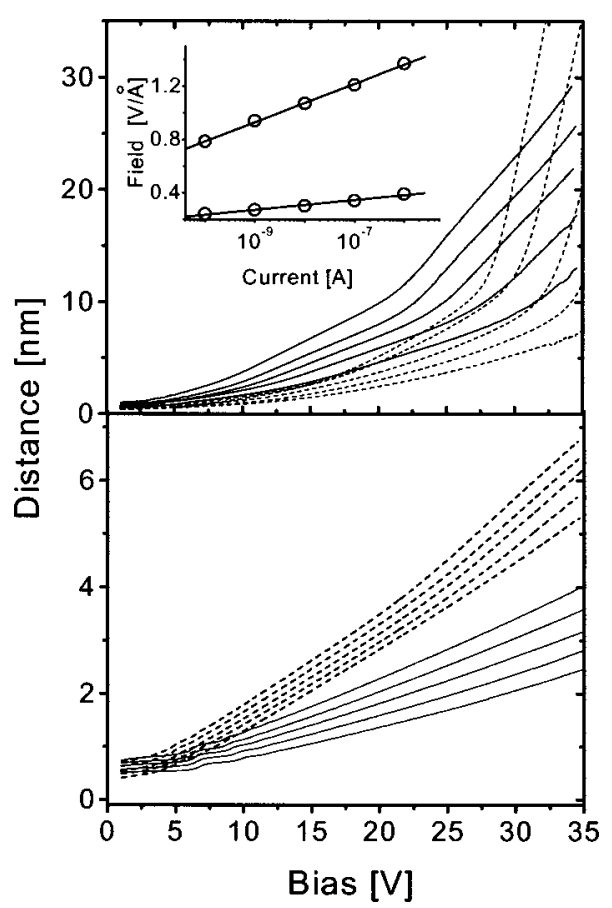

FIG. 2. $S(V)$ curves for constant field emission currents (top to bottom) of $100 \mathrm{pA}, 1 \mathrm{nA}, 10 \mathrm{nA}, 100 \mathrm{nA}$, and $1 \mu \mathrm{A}$. (a) Au (dashed lines) and Dy (solid lines); (b) W (dashed lines) and Pt (solid lines). In all cases voltage sweep started at $\sim 1.0 \mathrm{~V}$. Inset in (a) shows the electric field strength at the surface of the collecting electrode as a function of the field emission current.

and the shape of the electrodes. Typical sets of $S(V)$ curves taken at different values of the current $I=$ const, ranging from $100 \mathrm{pA}$ to $1 \mu \mathrm{A}$, are shown in Fig. 2(a) for Dy and $\mathrm{Au}$ and in Fig. 2(b) for Pt and W.

In the near-field emission regime $(e V \gtrsim \phi)$ the electric field at the surface of both the emitter and collector electrodes is described by $F_{e} \approx F_{c} \approx V / S$. In the following we assume the negatively biased emitting electrode to be sharper than the collecting one in order to preserve the "tip-sample" analogy (MCBJ as STM). This discrimination can easily be done from $S(V)$ curves measured for both polarities. ${ }^{17}$ The field on the apex of the emitter electrode is given by $F_{e}$ $\approx F_{c}(S+R) / R,{ }^{18}$ where $R$ is the tip radius. Then

$$
S(V) \approx \frac{V}{F_{e}}\left(1-\frac{V}{F_{e} R}\right)^{-1} .
$$

However, the radius of curvature of the emitting electrode is not constant in most cases (especially for ductile metals) which results in a more complex behavior of distancevoltage characteristics. For example, $S(V)$ curves for Au and Dy can be approximated by Eq. (1) only in a limited range $(\$ 7 \mathrm{~nm})$ of electrode separations [Fig. 2(a)]. Rapid increase in $S(V)$ for Au above $25 \mathrm{~V}$ is related to the relatively small radius and corrugation at the scale $\leqslant 10 \mathrm{~nm}$ of the surface of the electrodes. ${ }^{16,18}$ In contrast, the effective radius of the $\mathrm{Pt}$ and $\mathrm{W}$ electrodes exceeds few hundred nanometers and makes the situation close to two-dimensional [Fig. 2(b)].

The electric field strength at the surface of electrodes can be found from the difference $V_{21}$ of the positions of the first two peaks in the FER spectra as proposed in Ref. 19. Such spectra are presented in Figs. 3(a) and 3(b) for Dy and Pt, 


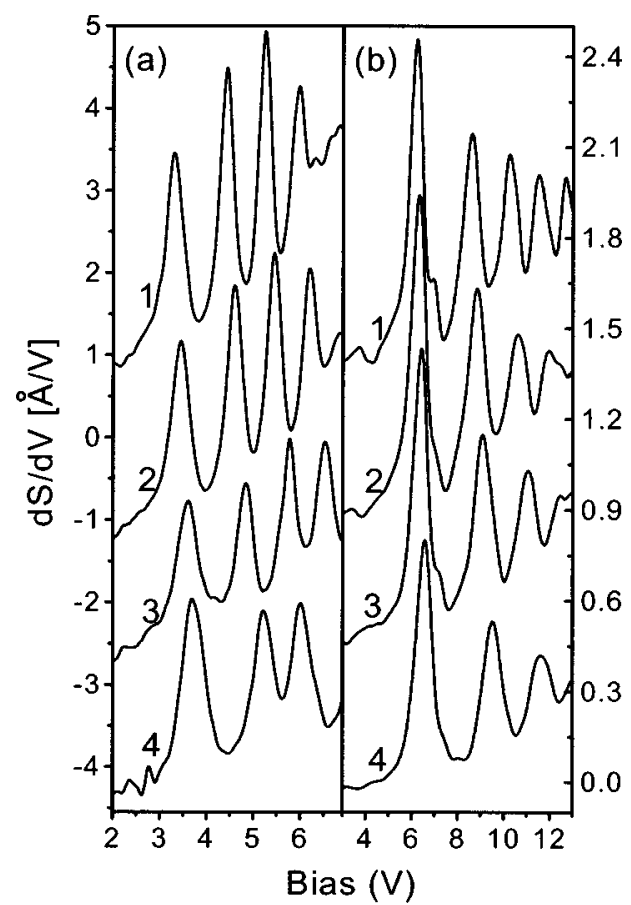

FIG. 3. Field emission resonance spectra for Dy (a) and Pt (b) for field emission currents of $100 \mathrm{pA}$ (curve 1), $1 \mathrm{nA}$ (curve 2), $10 \mathrm{nA}$ (curve 3), and $1 \mu \mathrm{A}$ (curve 4). Note a peak shift to higher bias voltages and an increase of the separation between neighboring peaks for an increasing current.

respectively. The shift of the peaks to a higher voltage as well as the increase of the separation between two neighboring peaks for an increasing field emission current are clearly observed. Taking into account that the eigenstates of the asymmetric triangle potential are expressed as

$$
E_{n}=\left(\frac{\hbar^{2}}{2 m}\right)^{1 / 3}\left[\frac{3 \pi F}{2}\left(n-\frac{1}{4}\right)\right]^{2 / 3}, \quad n=1,2, \ldots,
$$

and substituting the interval $E_{2}-E_{1}$ by $V_{21}$ one can find the electric field strength. In this range of bias voltages (electrode separations) it is approximately the same for both electrodes.

The inset in Fig. 2(a) shows the electric field strength at the surface of the Dy and Au electrodes as a function of the field emission current calculated from the relation

$$
F_{e, c}=V_{21}^{3 / 2} / 4.56,
$$

where $F_{e, c}$ is in $\mathrm{eV} / \AA$ and indices $e$ and $c$, respectively, refer to the emitter and collector electrodes. The electric field strength is proportional to $\ln I$ and increases nearly by a factor of 2 as the current $I$ increases from $100 \mathrm{pA}$ to $1 \mu \mathrm{A}$. Calculation of the first eigenstate $E_{1}$ using $F_{e}$ [Eq. (2)] allows us to find the zero energy level $E_{0}=V_{1}-E_{1}$. In the case of $\mathrm{Au}, E_{0} \simeq 5.2 \mathrm{eV}$ which is in good agreement with the work function of gold.

In the case of gold MCBJ an increase of the feedback (field emission) current up to $\approx 3-5 \mu \mathrm{A}$ eventually results in the threshold field that initiates the field-induced surface modification process. It should be noted, however, that at this current level the dissipated power reaches $10^{-4} \mathrm{~W}$ and the thermal effects become unavoidable. At a separation of about $3.5 \AA$ between the electrodes and a bias voltage of $5 \mathrm{~V}$ the

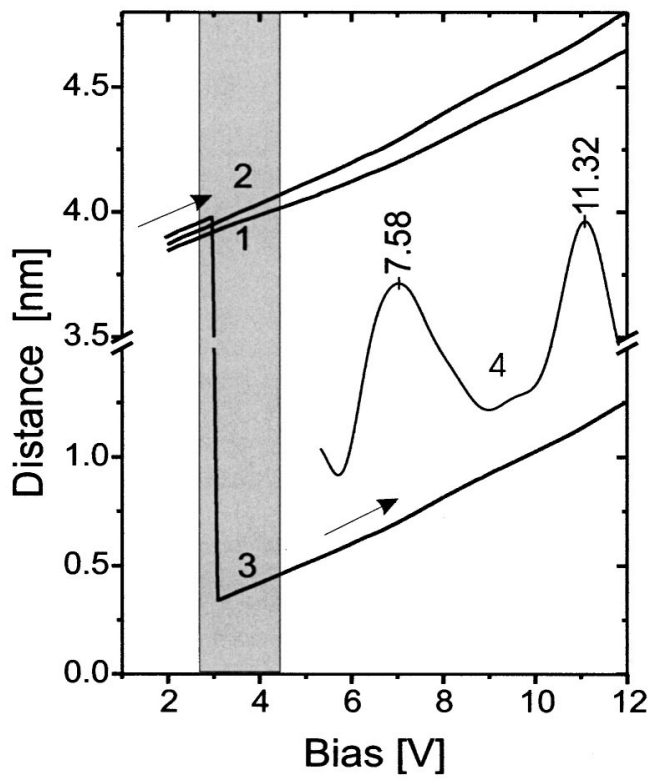

FIG. 4. Field evaporation from the surface of a gold electrode. $S(V)$ curves are presented for field emission currents of 1.5 (curve 1), 2.5 (curve 2), and $3 \mu \mathrm{A}$ (curve 3 ). The calculated electric-field strength for curve 2 is $1.6 \mathrm{~V} / \AA$. The shadowed box indicates the range of bias voltages where the surface modification process takes place. Curve 4-initial part of FER spectrum.

current density reaches $10^{8} \mathrm{~A} / \mathrm{cm}^{2}$. The overheating of the surface layer within the inelastic mean free path of hot electrons (estimated to range from 1 to $10 \mathrm{~nm}$ ) can be on the order of a few hundred degrees. ${ }^{20}$ However, the temperature of the electrode's bulk remains much lower and ensures the overall stability of the contact. Although the temperature of the electrode surface might be of a crucial significance for the initiation of field evaporation, we found no indications that a direct contact between the electrodes is formed on a time scale up to $10 \mu \mathrm{s}$ (cutoff frequency of our current-tovoltage converter)

A typical set of $S(V)$ curves for the evaporation field measurements of Au is presented in Fig. 4. These distancevoltage dependencies were measured for field emission currents from 1 to $3 \mu \mathrm{A}$ with a step of $0.5 \mu \mathrm{A}$. For the sake of clarity only three such curves are shown in Fig. 4. The curve for $I=3 \mu \mathrm{A}$ demonstrates an abrupt increase of the distance $\Delta S$ between the electrodes of $\approx 3.5 \mathrm{~nm}$ around a bias voltage of 3 V. (In Fig. 4 it looks like a sudden decrease in distance due to reaction of a feedback circuit reestablishing a designated current value). The value of $F_{e}$ found from the FER spectrum of $S(V)$ for $I=2.5 \mu \mathrm{A}$ was $\simeq 1.6 \mathrm{~V} / \AA$. We found that the interval of electric field strength at which field evaporation of gold occurred was always between 1.5 and $1.7 \mathrm{eV} / \AA$. This scattering of data is explained by the irregular shape of the Au electrodes and, therefore, the difference between the actual $F_{e, c}$ and the calculated one. The corresponding range of bias voltages was restricted to $3-4.5 \mathrm{~V}$ (shadowed part in Fig. 4). In general, $\Delta S$ was found to be mostly in an interval of $0.5-10 \mathrm{~nm}$.

The value of the evaporation field in our experiments is very close to that calculated for gold, ${ }^{21}$ but approximately three to four times larger than $F_{c}$ found in modification ex- 
periments with voltage pulses. ${ }^{5,6,22}$ At the same time, we observed the surface modification process in the bias voltage interval which practically coincides with the threshold voltage found for gold in earlier experiments. This discrepancy (an apparently lower field, the same bias voltage) can be explained in the following way: The tip-sample separation in experiments with voltage pulses decreases due to thermal expansion of the electrodes. As a consequence, the actual electric field strength is higher than estimated for the initial tip position. It should be noted that, once started, the process of electrode thermal expansion gradually takes on an avalanchelike character. Therefore, the outcome of the applied voltage pulse-field evaporation of metal or direct mechanical contact-depends on the competition between the two processes and is determined by a number of parameters, including pulse amplitude and its duration, the initial electrode separation, their shape, geometrical dimension, work function of material, etc.

The value of $F$ for a given emission current is determined by the work function of the electrode, $F \propto \phi^{3 / 2}$. Therefore, at the same emission current $F$ is essentially larger for such metals as gold (benchmark material in the surface modification experiments) with $\phi \simeq 5.3 \mathrm{eV}$ than for, e.g., Dy with $\phi \simeq 2.8 \mathrm{eV}$ [see inset in Fig. 2(a)]. Therefore, a sufficiently high electric field strength $(\geqslant 1 \mathrm{~V} / \AA)$ can be reached at acceptable power dissipation only for metals with large work function. In addition, to clearly observe the field induced effects the evaporation field must be sufficiently low. Although for Pt the work function $\phi \simeq 5.5 \mathrm{eV}$, it is difficult to observe the field induced modification process as the evaporation field for this metal is about $3 \mathrm{~V} / \AA^{21} .{ }^{21}$ In order to reach this field, the emission current should be $\gg 10 \mu \mathrm{A}$. Then, the temperature effects start to dominate the junction behavior (see Sec. III B).

In our earlier work ${ }^{23}$ we found that physical adsorption of He on surfaces drastically (by almost a factor of 2) increases the work function of all metals investigated so far. A similar result was reported in Ref. 24 where the interatomic distance $d$ in a single-atom gold chains was calibrated. In $\mathrm{He}$ exchange gas the authors found $d$ equal to $3.6 \AA$, whereas UHV measurements resulted in the theoretically predicted $2.5 \AA$. This disagreement can be explained by assuming an increase in work function of $\mathrm{Au}$ due to $\mathrm{He}$ adsorbtion by a factor of 1.8. We used this effect to enhance the electric field strength in our MCBJ experiments. In Fig. 5 our results for tungsten are presented. Modification of a He covered surface occurs at extremely low emission currents ranging from 1 to $100 \mathrm{pA}$. Typically, the power dissipated in electrodes is less than $10^{-6} \mathrm{~W}$ and the thermal effects are vanishingly small. Depending on the metal and the field emission current $S(V)$ curves start to exhibit a nonmonotonic behavior at $V_{b}$ $\simeq 10-15 \mathrm{~V}$. A further increase of the bias voltage results in visible jumps of the interelectrode separation at $V_{b} \geqslant 25 \mathrm{~V}$ and, finally, in an apparent decrease of the distance between the electrodes. Reversing the direction of the voltage scan and arriving at the starting $V_{b}$, we observed a shift of the $S(V)$ dependence down on the distance scale by few tens of nanometers. This distance is four orders of magnitude larger than the expected electrode drift and indicates a decrease of

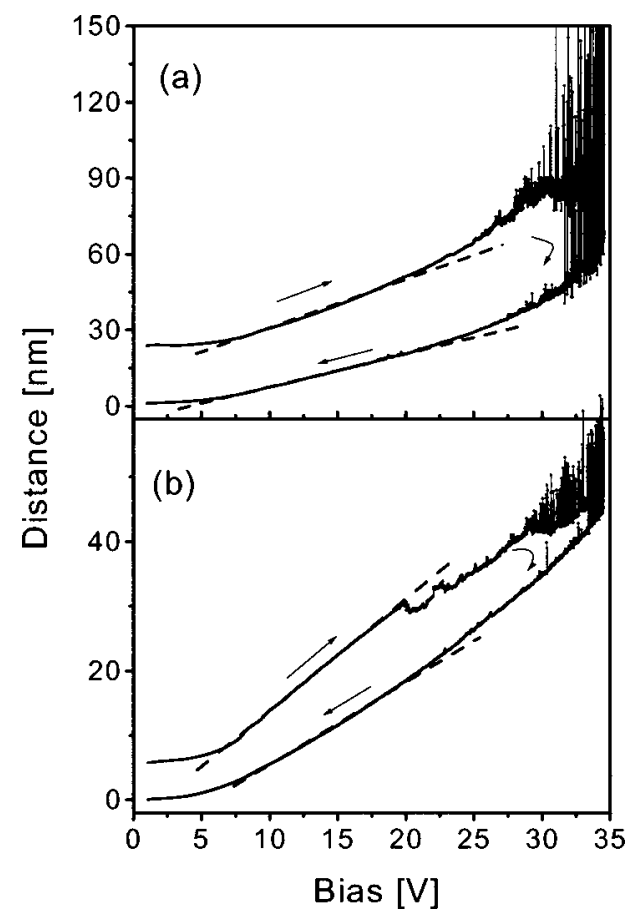

FIG. 5. The modification process on He-covered metallic surface for tungsten MCBJ. The $S(V)$ curve in (a) was taken for a feedback current of $10 \mathrm{pA}$ in He exchange gas and in (b) in liquid He with a feedback current of $30 \mathrm{pA}$.

the electrode length. Moreover, the linear part of the $S(V)$ curves becomes less steep (dashed lines in Fig. 5) due to an increase of the electrode radius (blunting of the electrode surface).

To explain the above phenomena we propose the following model. At the first stage of the modification process, adsorbed $\mathrm{He}$ is removed from the electrode surface. At the second stage, the field evaporation/desorption of metallic atom(s) occur from the "bare" part of the surface. Finally, adsorption of He from the gaseous or liquid phase (or surface diffusion of $\mathrm{He}$ ) restores the initial conditions. In a range of bias voltages between 10 and $25 \mathrm{~V}$ the main mechanism behind $\mathrm{He}$ desorption is electron-stimulated field desorption (by electron impact adatoms are ionized and instantly field desorbed). This effect can be clearly observed at higher field emission currents as the required incoming electron rate must be $\gtrsim 10^{5} \mathrm{e} / \mathrm{s}$. At bias voltages above $25 \mathrm{~V}$ desorption of helium atoms occurs also as a result of a field ionization. ${ }^{25}$ It should be noted that in electric field of 3-4 V/ $\AA$ the adsorption potential of $\mathrm{He}$ remains very shallow $(\simeq 5-10 \mathrm{meV}) .{ }^{26}$ For even larger voltages $\mathrm{He}$ atoms can be temporarily removed from a sizable part of the surface. The work function of the bare metal is significantly lower than that of a He-covered surface. This leads to an abrupt increase of the emission current and forces the feedback system to retract the electrodes [spikes in the $S(V)$ characteristics (Fig. 5)]. The intensity of the modification process increases with increasing $V_{b}$, resulting in field evaporation of ever larger portions of the electrodes. For extremely large bias voltages, $V_{b} \gtrsim 35 \mathrm{~V}$, the amount of the field evaporated material is so large that the feedback system cannot be corrected for it and the electrodes are out of reach. Surface modification in gaseous He [(Fig. 5(a)] is faster than in liquid He [(Fig. 5(b)] as 
the desorbed $\mathrm{He}$ atoms are replaced faster from the liquid phase. In superfluid He we observed additional moderation of this modification process.

Although the model proposed above provides only a very simplified picture, it explains the main experimental observations. Additionally, some direct analogies with physical processes in a field ion microscope (FIM) can be traced down. In those FIM experiments an auxiliary gas (e.g., $\mathrm{H}_{2}$ ) with a low ionization energy was used as a source of electrons. It was also shown ${ }^{27}$ that desorption of $\mathrm{He}$ atoms occurs primarily from the protruding parts of the surface. This explains the "blunting" of the electrode surface.

We note here that observation of FER spectra for He-covered metallic surfaces is rather challenging. For the specific case of He covered W (discussed above) we found a work function of roughly $7.5 \mathrm{eV}$ and an electric field strength of $1.2-1.4 \mathrm{~V} / \AA$. This field is significantly lower (by about a factor of 2) than that calculated in Ref. 21. However, it is not quite clear to us to what extent the use of Eq. (2) in our analysis of He-covered surfaces is justified (see also Sec. III B). ${ }^{28}$

We found that all metals investigated in our experiments, including Dy with an extremely low work function, can be modified in a He environment. The modification process can be controlled carefully by adjustment of the emission current, the voltage scan rate, and the He pressure.

\section{B. Temperature effects}

For materials with high evaporation fields measured in UHV the increase of the field emission current eventually results in conditions where temperature starts to play a crucial role. It is possible to accurately trace this transition in W-MCBJ. Because of a high melting point and strong bonds between tungsten atoms the measurement is still possible, despite the fact that the power dissipated in the electrodes reaches values as high as $10^{-3} \mathrm{~W}$. At the same time, we were not able to obtain field values exceeding $1.2-1.3 \mathrm{~V} / \AA$ even for an emission current of $100 \mu \mathrm{A}$. (Note that in a He environment we were able to reach such fields at current seven to eight orders of magnitude smaller.) For these high current levels the thermal elongation of the electrodes $\Delta S(T)$ is on the same order of magnitude as the bias related part of the $S(V)$ dependence. Elevated electrode temperatures result in an instability of the FER spectrum-original spectral peaks are smeared and spurious peaks that are related to the irregular thermal motion of the electrode surfaces appear.

We measured thermal expansion of the electrodes in a constant voltage mode by scanning the tunnel/emission current and observing the feedback reaction. For that we assumed that the distance between the anchoring points remains unchanged in the course of the experiment. In Fig. 6 four $S(I)$ curves for fixed bias voltages of 1 and $7 \mathrm{~V}$ [Fig. 6(a)] and 15 and $25 \mathrm{~V}$ [Fig. 6(b)] are presented. For small current values the feedback pushes the electrodes together as the current is increased [inset in Fig. 6(b)]. However, for larger currents thermal expansion of the electrodes starts to dominate and forces the feedback to retract [see inset in Fig. 6(a)]. The $S(I)$ curves in Fig. 6(a) demonstrate a linear de-

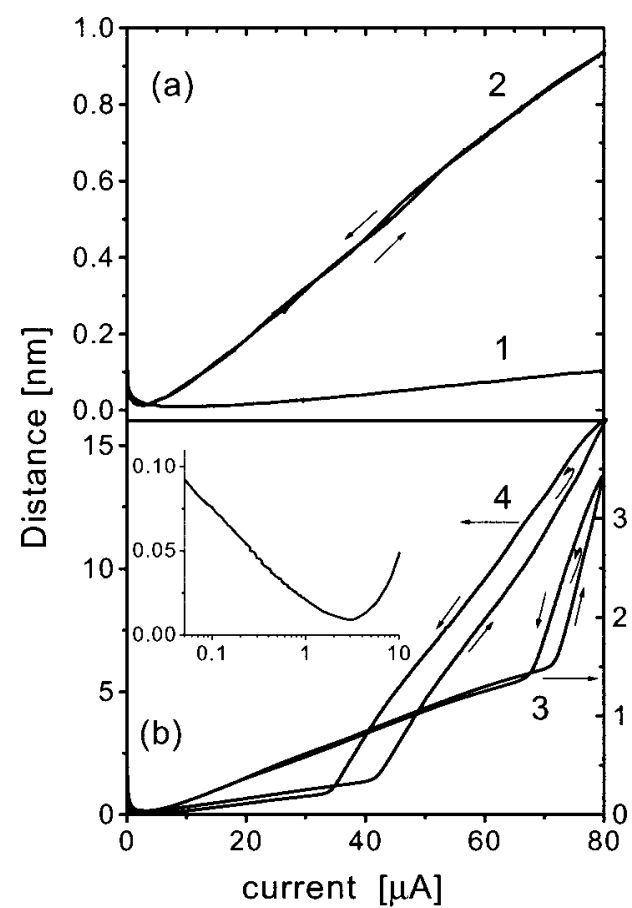

FIG. 6. Dependence of the electrode elongation $\Delta S(I)$ on the field emission current for fixed bias voltages. Curves 1 and 2 (a) measured in the tunneling $\left(V_{b}=1 \mathrm{~V}\right)$ and near-field $\left(V_{b}=7 \mathrm{~V}\right)$ regimes show a linear increase of $\Delta S(I)$, while curves measured for higher bias voltages of $15 \mathrm{~V}$ (curve 3 ) and $20 \mathrm{~V}$ (curve 4) are nonlinear due to changes of the heat removal conditions. Inset in (b) shows the initial part of the $\Delta S(I)$ curve at $V_{b}=4 \mathrm{~V}$.

pendence of the electrode elongation on the emission current or, equivalently, on the dissipated power. The estimated overheating of the electrode's bulk, which is derived from an elongation of $\simeq 1.5 \mathrm{~nm}$, does not exceed $100 \mathrm{~K}$. However, the temperature of the surface layer with a thickness on the order of the electron inelastic mean free path can reach more than $1000 \mathrm{~K}^{20}$ The curves in Fig. 6(b) exhibit a sharp transition to a nonlinear behavior of the electrode elongation and show a pronounced hysteresis. We attribute this effect to abrupt changes of the heat removal conditions at elevated temperatures (e.g., the temperature-dependent heat conductivity of hard epoxy and piezoceramic plates can exhibit some anomalies).

We performed additional time resolved measurements of the elongation of the tungsten electrode. To this end we switched the field emission current from 0.1 to $70 \mu \mathrm{A}$ and measured the time-dependent electrode retraction. A typical $\Delta S(t)$ dependence taken for a bias voltage of $10 \mathrm{~V}$ is presented in Fig. 7 . The data below $\approx 0.5 \mathrm{~ms}$ should be disregarded because of the transient processes in the feedback circuit. For longer times, the electrode elongation is proportional to $t^{1 / 2}$. This dependence has a different slope during the initial stage up to $10 \mathrm{~ms}$ and in the interval from $\sim 15$ to $300 \mathrm{~ms}$. The later stage corresponds to a slower heating rate of the unglued part of the electrode due to heat dissipation via the electrical leads and anchoring points to the massive bending beam. The overall behavior of $\Delta S(t)$ dependence is very similar to our earlier measurements of the electrode elongation in the tunnel regime, ${ }^{13}$ although at the present level of the dissipated power overheating of the electrode is 


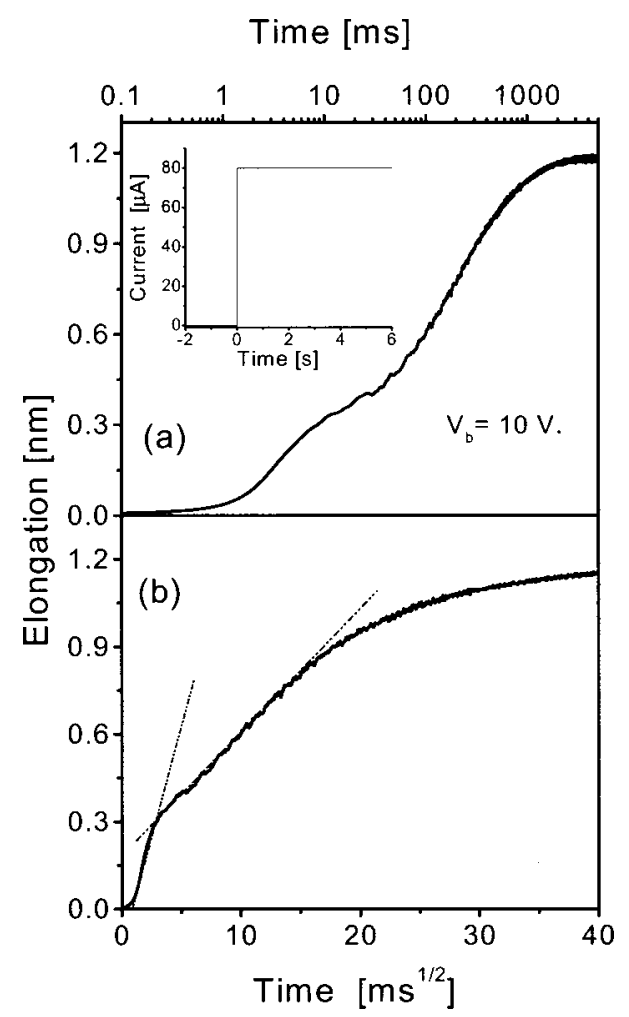

FIG. 7. Logarithmic plot of $\Delta S(t)$ for W-MCBJ (a) and the $\Delta S\left(t^{1 / 2}\right)$ dependence for the same junction (b). Inset: feedback current diagram.

one order of magnitude higher. Larger values of thermal conductivity and linear thermal expansion coefficient at higher temperatures (our present experiment versus Ref. 13) result in an electrode elongation almost two orders of magnitude larger and on a shorter time scale.

\section{CONCLUSIONS}

In conclusion, we presented our experiments on surface modification in MCBJ. By using advantages of this technique, primarily the intrinsic junction stability, we could separate the electric field induced effects from those caused by the rising temperature. Clear observation of field evaporation/desorption in both the tunnel and near-field emission regimes is possible only for a limited number of metals with large work functions and/or sufficiently low evaporation fields. A twofold increase of the work function by adsorbed $\mathrm{He}$ enabled us to control the modification of all metallic surfaces studied so far. The model proposed here includes field desorption of $\mathrm{He}$ followed by immediate desorption/evaporation of metallic atoms. Finally, the exceptional properties of tungsten permitted us to study its thermal expansion on a nanometer scale for extremely high emission currents.

\section{ACKNOWLEDGMENTS}

The authors are grateful to A. Toonen, J. Hermsen, and J. Gerritsen for invaluable technical assistance. Part of this work was supported by the Stichting voor Fundamenteel Onderzoek der Materie (FOM) which is financially supported by the Nederlandse Organisatie voor Wetenschappelijk Onderzoek (NWO). The Hungarian Research Funds (OTKA Grant Nos. TO37451, TS040878, and N31769) and a NWO grant for Dutch-Hungarian cooperation are also acknowledged. O.I.S. wishes to acknowledge the NWO for a visitor's grant.

${ }^{1}$ J. Tersoff and D. R. Hamman, Phys. Rev. Lett. 50, 1998 (1983).

${ }^{2}$ U. Staufer, in Scanning Tunneling Microscopy II, Springer Series in Surface Sciences, Vol. 28, edited by R. Wiesendanger and H.-J. Güntherdot (Springer, Berlin, 1992).

${ }^{3}$ R. Kóning, O. Jusko, L. Koenders, and A. Schlachetzki, J. Vac. Sci. Technol. B 14, 48 (1996).

${ }^{4}$ T. M. Mayer, J. E. Houston, G. E. Franlin, A. A. Erchak, and T. A. Michalske, J. Appl. Phys. 85, 8170 (1999).

${ }^{5}$ H. J. Mamin, P. H. Guethner, and D. Rugar, Phys. Rev. Lett. 65, 2418 (1990).

${ }^{6}$ C. S. Chang, W. B. Su, and T. T. Tsong, Phys. Rev. Lett. 72, 574 (1994).

${ }^{7}$ X. Hu and P. von Blanckenhagen, J. Vac. Sci. Technol. B 17, 265 (1999).

${ }^{8}$ J. I. Pascual, J. Mendez, J. Homes-Herrero, A. M. Baro, N. Garcia, and Vu T. Binh, Phys. Rev. Lett. 71, 1852 (1993).

${ }^{9}$ G. G. Vladimirov and A. V. Drozdov, J. Vac. Sci. Technol. B 15, 482 (1997).

${ }^{10}$ G. G. Vladimirov, A. V. Drozdov, and L. M. Baskin, Tech. Phys. Lett. 21, 426 (1995).

${ }^{11}$ O. Hansen, J. T. Ravnkilde, U. Quaade, K. Stokbro, and F. Grey, Phys. Rev. Lett. 81, 5572 (1998).

${ }^{12}$ O. Yu. Kolesnychenko, O. I. Shklyarevskii, and H. van Kempen, Rev. Sci. Instrum. 70, 1442 (1999).

${ }^{13}$ O. Yu. Kolesnychenko, A. J. Toonen, O. I. Shklyarevskii, and H. van Kempen, Appl. Phys. Lett. 79, 2707 (2001).

${ }^{14}$ J. M. van Ruitenbeek, in Metal Cluster on Surfaces: Structure, Quantum Properties, Physical Chemistry, edited by K. H. Meiwes-Broer (Springer, Heidelberg, 2000), pp. 175-210; N. Agrait, A. Levy Yeyati, and J. M. van Ruitenbeek, Phys. Rep. 377, 81 (2003).

${ }^{15}$ R. S. Becker, J. A. Golovchenko, and B. S. Swartzentruber, Phys. Rev. Lett. 55, 987 (1985).

${ }^{16}$ J. H. Coombs and J. K. Gimzewski, J. Microsc. 152, 841 (1988).

${ }^{17}$ O. Yu. Kolesnychenko, O. I. Shklyarevskii, and H. van Kempen, Physica B 284, 1257 (2000).

${ }^{18}$ G. Mesa and E. Sáenz, Appl. Phys. Lett. 69, 1169 (1996); G. Mesa, E. Dobado-Fuentos, and J. J. Sáenz, Jpn. J. Appl. Phys., Part 2 79, 39 (1996).

${ }^{19}$ Y. Suganuma and M. Tomitori, Jpn. J. Appl. Phys., Part 1 39, 3758 (2000).

${ }^{20}$ F. Flores, P. M. Echenique, and R. H. Ritchie, Phys. Rev. B 34, 2899 (1986).

${ }^{21}$ T. T. Tsong, Phys. Rev. B 44, 13703 (1991).

${ }^{22}$ A. Ohi, W. Mizutani, and H. Tokumoto, J. Vac. Sci. Technol. B 13, 1252 (1995).

${ }^{23}$ O. Yu. Kolesnychenko, O. I. Shklyarevskii, and H. van Kempen, Phys. Rev. Lett. 83, 2242 (1999).

${ }^{24}$ C. Untiedt, A. I. Yanson, R. Grande, G. Rubio-Bollinger, N. Agraït, S. Vieira, and J. M. van Ruitenbeek, Phys. Rev. B 66, 085418 (2002).

${ }^{25}$ N. Ernst, Phys. Rev. Lett. 45, 1573 (1980).

${ }^{26}$ N. Ernst, W. Drachel, Y. Li, J. H. Block, and H. J. Kreuzer, Phys. Rev. Lett. 57, 2686 (1986)

${ }^{27}$ J. A. Panitz, J. Vac. Sci. Technol. 12, 210 (1974).

${ }^{28}$ A. J. Caamaño, Y. Pogorelov, O. Custance, J. Méndes, A. M. Baró, J. Y. Veuillen, J. M. Gómes-Rodriges, and J. J. Sáenz, Surf. Sci. 426, L420 (1999). 